\title{
SOLUBILIZATION OF TENOXICAM VIA DIFFERENT TECHNIQUES
}

\author{
M. I. Fetuoh", S. Ismail ${ }^{* *}$, S. A. El-Harras** and M. Abbas* \\ "Department of Pharmaceutics, Faculty of Pharmacy, Al-Azhar University \\ "* Department of Pharmaceutics, Faculty of Pharmacy, Assiut University, Egypt
}

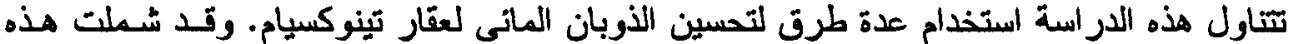

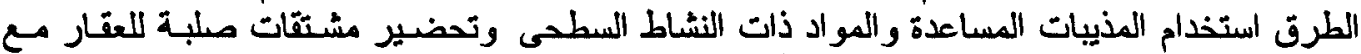

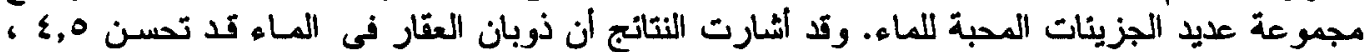

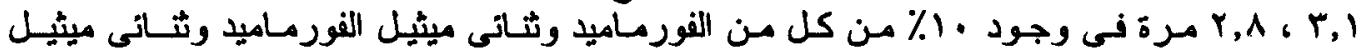

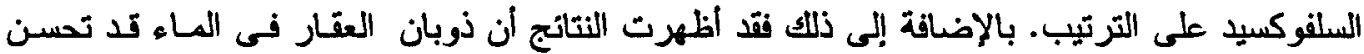

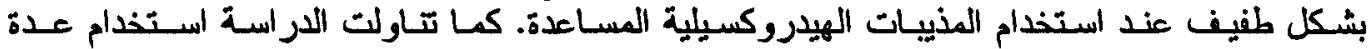

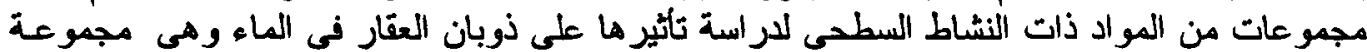

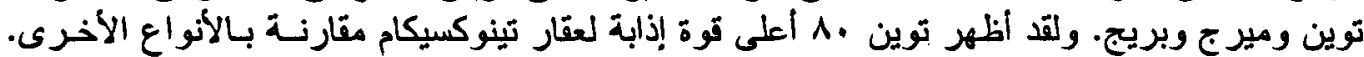

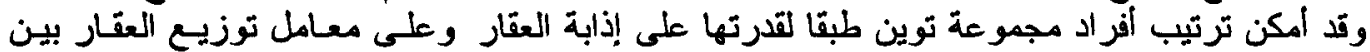

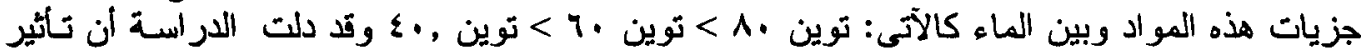

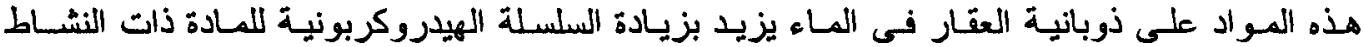

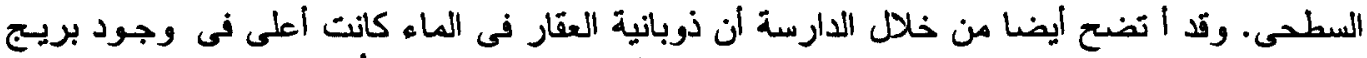

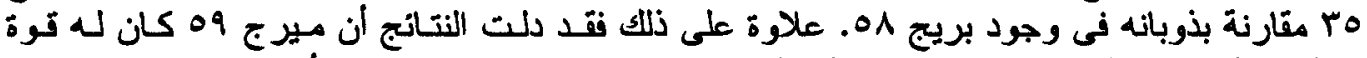

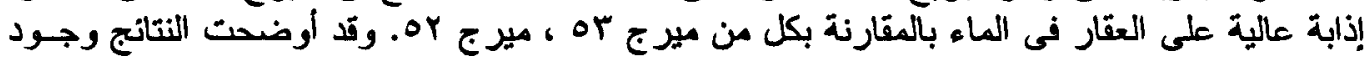

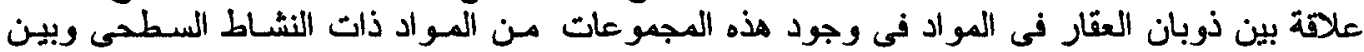

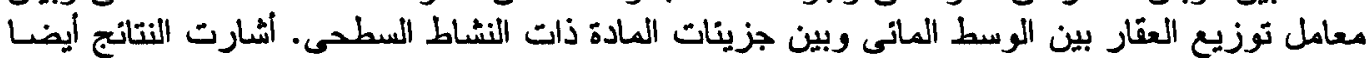

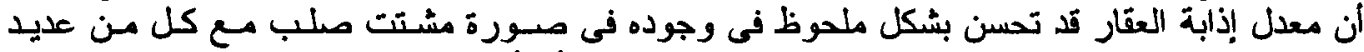

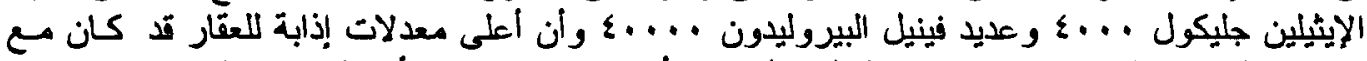

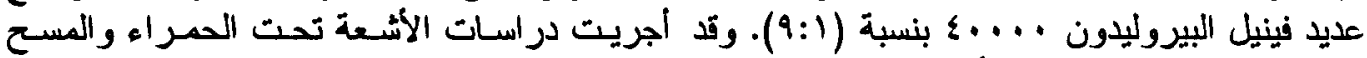

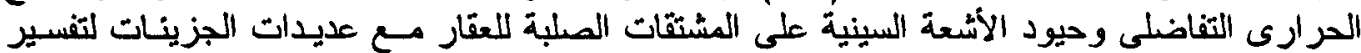

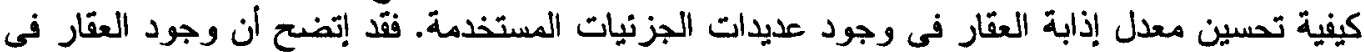

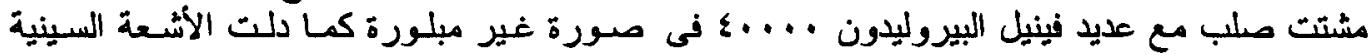

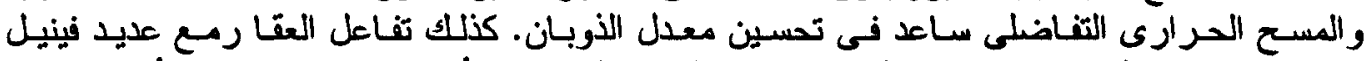

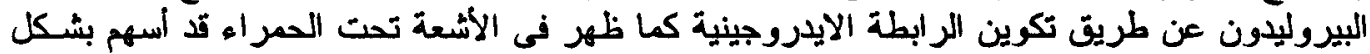

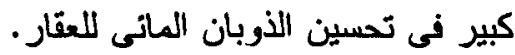

In this study, several techniques were employed to improve the aqueous solubility of tenoxicam. These techniques include co-solvency, micellar solubilization and solid dispersions using different water-soluble polymers. The aqueous solubility of tenoxicam in presence of $10 \%$ of formamide, DMF and DMSO was enhanced about 4.5, 3.1 and 2.8 times, respectively. In addition, the results show that the hydroxylated co-solvents (glycerol, PG, PPG, PEG 200, PEG 300, PEG 400 and PEG 600) just potentiated the aqueous solubility of the drug. Several classes of non-ionic surfactants were studied for their solubilizing action on tenoxicam. These classes were polysorbates (Tweens), polyoxyethylene alkylethers (Brijs) and polyoxyethylene stearates (Myrjs). Tween 80 exhibited a better solubilizing capacity on tenoxicam than other investigated tweens and they could be arranged according to their solubilizing capacity on the drug and the distribution coefficient of the drug between their micelles as: Tween $80>$ Tween $60>$ Tween 40. It is clearly evident that the solubilizing effect of these micellar-forming agents increases as the hydrophobic chain length of the surfactant increases. In addition, Brij 35 exhibited a higher solubilizing capacity on the drug than Brij 58. Furthermore, Myrj 59 exhibited higher solubilizing power on tenoxicam compared to Myrj 53 and Myrj 52. These results indicate the relationship between the aqueous solubility of the drug in presence of the 
above tested surfactants and its distribution coefficient between micellar and aqueous phases. The dissolution rate of tenoxicam in presence of PEG 4000 and PVP 40000 in its solid dispersions was improved especially with PVP 40000 (in a drug: polymer ratio 1:9). IR, DSC and $X$-ray diffraction studies were conducted to investigate the mechanism responsible for this improvement.

\section{INTRODUCTION}

Tenoxicam is considered as a member of the non-steroidal anti-inflammatory drugs (NSAIDs) which used in treatment of several rheumatic disorders as arthritis and rheumatoid arthritis. Chronic administration of this drug causes several side effects. These adverse effects include gastric ulceration and change in hepatic and renal functions. The problems associated with the frequent administration of this drug could be solved using alternative routes of administration. This could be achieved by formulating the drug in topical dosage forms. The problem facing the formulation of tenoxicam in topical preparations is its lower aqueous solubility.

Several techniques are employed to improve the aqueous solubility of drugs. These techniques include cosolvency, micellar solubilization and solid dispersion technique.

A wide variety of poorly soluble drugs could be solubilized using water miscible solvents as glycerol, propylene glycol, ethanol, dimethyl formamide, etc.... Shawesh et al. ${ }^{1}$ studied the aqueous solubility of indomethacin in different co-solvents. They observed that a higher solubility of the drug was attained in presence of $80 \%$ ethanol.

In topical formulations, some co-solvents are widely used not only to increase drug solubility, but also used to enhance penetration. $^{2,3}$

Surface active agents at the concentration above CMC are most widely used as means of producing aqueous solutions of insoluble or poorly soluble drugs. Numerous studies of various compounds in different non-ionic surfactants solutions were reported. ${ }^{48}$

Solid dispersions were introduced in the early $1960_{\mathrm{s}}{ }^{9}$ and since then a variety of insoluble drugs have been formulated as solid dispersions. ${ }^{10-13}$ The term solid dispersion refers to the dispersion of one or more active ingredients in an inert matrix at solid state. ${ }^{14}$
The matrix is composed of high molecular weight water soluble polymer(s). ${ }^{12}$

Thus, the purposes of this work were: 1) To improve the aqueous solubility of tenoxicam in presence of different concentrations of certain cosolvent systems viz., formamide, dimethyl formamide (DMF) and dimethyl sulfoxide (DMSO). 2) To study the solubilization of this drug using series of nonionic surfactant solutions. The effect of variations in surfactant molecular structure on the degree of solubilization of the drug was investigated. 3) Preparation of solid dispersion of the drug using both PEG 4000 and PVP 40000 to enhance the in-vitro dissolution of tenoxicam. IR, DSC and x-ray diffraction were used to investigate the solid state physical structure of the drug -PEG and PVP solid dispersion systems.

\section{EXPERIMENTAL}

\section{Materials}

Tenoxicam, [Egyptian International Pharmaceutical Industries Co., Egypt]. Dimethyl formamide [DMF], formamide, and Tweens: Tween 40, 60\& 80 (BDH chemical ltd. Co., Poole, England). Dimethyl sulfoxide [DMSO], Propylene glycol [PG] and polypropylene glycol [PPG] (Aldrich chemical Co., England). Glycerol (EL-Nasr Co., AbuZabal, Egypt). Polyethylene glycols: PEG 200, PEG 300 and PEG 4000, (Fluka chemica, Buch, Switzerland). Polyethylene glycol PEG 400 (Merck Co., Germany). Myrjs: Myrj52, 53\&59, Brijs: Brij 35\&Brij 58 and Polyvinylpyrrolidone PVP 40000, (Sigma chemical Co., USA). Other materials and solvents are of reagent or analytical grade, and they were used without further purification.

\section{Methodology}

Effect of different additives on tenoxicam aqueous solubility

Solubility of the drug in water in presence of different concentrations of additives 
(Formamide, DMF, DMSO, glycerol, PG, PPG, PEG 200, 300, 400 and 600) was studied. In addition, the solubility in presence of different concentrations of non-ionic surfactants (Tween $40,60 \& 80$, Myrj 52, $53 \& 59$ and Brij $35 \&$ 58) was also studied. Solubility was studied using the following procedure: an excess amount of tenoxicam powder was added to $10 \mathrm{ml}$ of each solution in a $50 \mathrm{ml}$ glass stoppered bottle. The bottles were firmly closed and placed into the mechanical shaking water bath (SBS Instruments, Germany) previously adjusted at $37^{\circ} \pm 0.1$. After equilibration has been attained ( 6 hrs), one $\mathrm{ml}$ aliquot sample was withdrawn by a pipette fitted with a cotton plug at its terminal end from each tested solution and diluted to an appropriate volume with distilled water. The absorbance was measured at $371 \mathrm{~nm}$ (UV. 1601Shimadzu Co., Japan) against a suitable blank similarly treated and the drug concentration was calculated.

Preparation and characterization of tenoxicam dispersions with different watersoluble polymers

Two water-soluble polymers were employed for preparing both solid dispersions of tenoxicam and its corresponding physical mixtures. The polymers were PEG 4000 and PVP 40000.

\section{Preparation of tenoxicam solid dispersions}

Co-evaporation technique was employed to prepare solid dispersions of the tenoxicam with the different carriers, since fusion method can't be applied because the drug decomposes before it reaches its melting point.

Different drug: polymer ratios $(4: 1,3: 2$, $2: 3,1: 4$, and 1:9) were used for preparing solid dispersions of tenoxicam with the different polymers. The calculated amounts of tenoxicam and the polymer were dissolved in a minimum amount of chloroform. The solvent was allowed to evaporate at room temperature and the residue was dried at $40^{\circ}$ in a hot air oven for 24 $\mathrm{hr}$. The dried residue was placed in a dessicator containing anhydrous calcium chloride for further $24 \mathrm{hr}$. The resulted precipitate was pulverized and sieved (Rx-86-1 Cole-Parmer Instrument Co., USA). The fraction of particle size range between 315-250 $\mu \mathrm{m}$ was collected and used in the experimental studies.
Physical mixtures of some selected drug: polymer ratios were prepared by simple mixing of the drug and the polymer.

\section{Dissolution rate studies}

The dissolution rate of the drug from the prepared solid dispersions and some selected physical mixtures as well as the untreated drug was studied using USP dissolution apparatus (SR11 6 Flask, Hanson Co., USA). Accurately weighed amounts equivalent to $90 \mathrm{mg}$ of tenoxicam were dispersed over the dissolution medium $(250 \mathrm{ml}$ saline phosphate buffer, $\mathrm{pH}=$ 7.4) at $37^{\circ} \pm 0.1$ which immediately stirred at 50 rpm. At appropriate time intervals, one $\mathrm{ml}$ sample was withdrawn by a pipette fitted with a cotton plug at its terminal end. The sample was diluted to a suitable volume with the buffer solution and the absorbance was measured at $371 \mathrm{~nm}$. Equal volume of fresh dissolution medium, prewarmed at $37^{\circ}$, was replaced into the dissolution medium so as to keep the volume of the dissolution medium constant.

The cumulative amount of the drug dissolved during the $\mathrm{n}^{\text {th }}$ sample $(\mathrm{Qn})$ was estimated by the following equation: ${ }^{15}$

$$
Q_{n}=C_{n} \cdot V+V_{s} \cdot \sum_{i=1}^{n-1} C_{i}
$$

Where $C_{n}$ is the measured concentration in the $\mathrm{n}^{\text {th }}$ sample, $\mathrm{V}$ is the volume of dissolution medium, and $V_{s}$ is the volume of sample.

\section{IR-spectroscopy}

IR spectra of certain tenoxicam solid dispersions and the corresponding physical mixtures as well as the untreated drug were done at a range $4000-400 \mathrm{~cm}^{-1}$ using $\mathrm{KBr}$ disk method (IR-476, Shimadzu Co., Japan). The samples were ground, mixed thoroughly with $\mathrm{KBr}$ and compressed at a pressure of 6 ton $/ \mathrm{cm}^{2}$ using Shimadzu SSp-10A IR compression machine.

\section{Differential scanning calorimetry (DSC)}

DSC studies were carried out on previously prepared tenoxicam solid dispersions in a certain drug: polymer ratio and the corresponding physical mixtures as well as untreated drug in order to determine the extent of crystallinity of the drug in presence of the tested polymers. 
Samples of about $5 \mathrm{mg}$ were accurately weighed and encapsulated into flat-bottomed aluminum pans with crimped-on lids. The scanning speed of $10^{\circ} / \mathrm{min}$ from $30^{\circ}$ to $250^{\circ}$ was used in presence of nitrogen at flow rate of 40 $\mathrm{ml} / \mathrm{min}$. The instrument was initially calibrated with pure indium.

\section{X-ray diffraction analysis}

The X-ray diffraction patterns of the powder samples were obtained using a Philips 1700 series diffractometer which is equipped with curved graphite crystal monochromater, automatic divergence slit and automatic controller PW/1710. The target used was $\mathrm{CuK} \alpha$ radiation operating at $40 \mathrm{KV}$ and $30 \mathrm{~mA}\left(\lambda_{\mathrm{k \alpha}}=\right.$ $1.5418 \AA)$. The system was calibrated using silicon disc and/or powder $\left(\mathrm{d}_{111}=3.1355 \AA\right)$ as an external standard. The diffraction patterns were achieved using continuous scan mode with $2 \theta^{\circ}$ ranging from $4^{\circ}$ to $60^{\circ}$. The output data achieved represented by $2 \theta, \mathrm{d} \AA$, intensities were determined via the microprocessor of the PW/1710.

\section{RESULTS AND DISCUSSION}

Figures 1-3 illustrate the effect of different concentrations of formamide, DMF, DMSO, glycerol, PG, PPG, PEG 200, PEG 300, PEG 400 and PEG 600 when they were used in concentrations up to $10 \%$ on the aqueous solubility of tenoxicam at $37^{\circ}$.

It is clearly obvious that there is an improvement in the aqueous solubility of the medicament in presence of these solubilizers. The solubility of the drug was affected by both the type and the concentration of the solubilizer used. The aqueous solubility of tenoxicam in presence of $10 \%$ of each of formamide, DMF and DMSO was enhanced about 4.5,3.1 and 2.8 times, respectively. Formamide exerts a higher solubilizing effect compared to DMF. For instant, the aqueous solubility of the medicament was enhanced to $0.4 \mathrm{mg} / \mathrm{ml}, 0.28$ $\mathrm{mg} / \mathrm{ml}$ in presence of $10 \%$ formamide and DMF, respectively. Thus, alkylation of formamide may decrease the solubilizing action. In addition, tenoxicam aqueous solubility was greatly enhanced in the presence of different concentrations of DMSO. However, DMSO exerts a slightly lower solubilizing action on the drug than DMF.

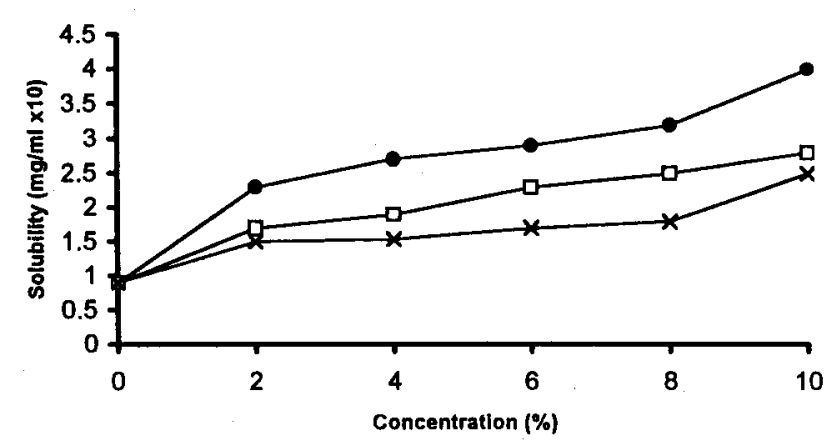

Fig. 1: Effect of Different Concentrations of Additives on Tenoxicam Aqueous Solubility at $37^{\circ}$. Key: •, Formamide; $\square$, DMF and $x$, DMSO.

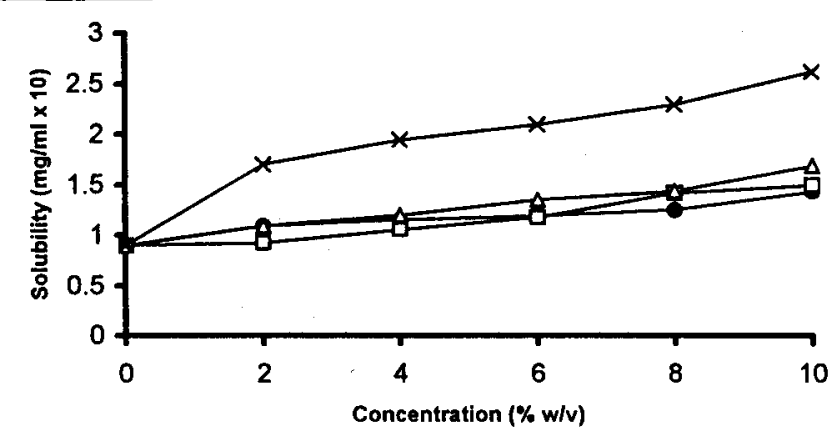

Fig. 2: Effect of Different Concentrations of Polyethylene Glycols (PEGs) on Tenoxicam Aqueous Solubility at $37^{\circ}$. Key: •, PEG 200; प, PEG 300; $\triangle$, PEG 400 and $x$, PEG 600 .

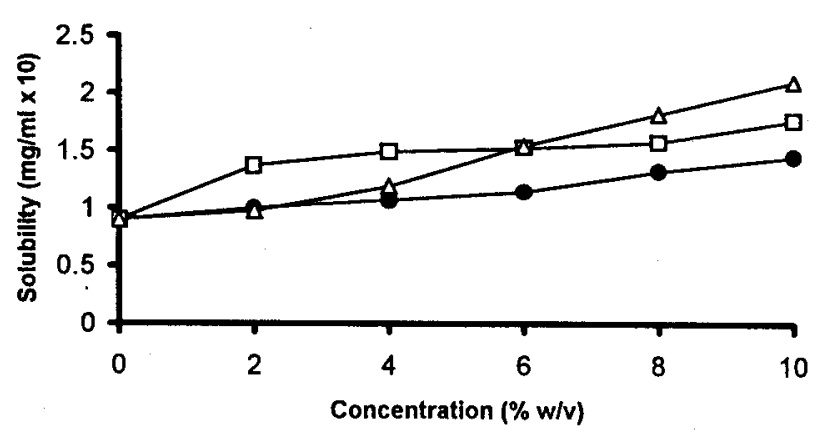

Fig. 3: Effect of Different Concentrations of Glycerol, PG and PPG on Tenoxicam Aqueous Solubility at $37^{\circ}$. Key: •, Glycerol; $\square$, PG and $\Delta$, PPG. 
The solubilizing effect of different hydroxylated co-solvents, viz. glycerol, propylene glycol (PG), polypropylene glycol (PPG) and polyethylene glycols (PEG 200, PEG 300, PEG 400 and PEG 600) on tenoxicam was investigated. These hydroxylated co-solvents were found to just enhance the drug aqueous solubility as shown in Figures $2 \& 3$.

On comparing the solubilizing effect of PEGs on the aqueous solubility of tenoxicam as shown Figure 2, PEG 600 shows the highest solubilizing action followed by PEG 400, PEG 300 and PEG 200. It is clearly evident that by increasing the chain length of PEG, the aqueous solubility of the drug was enhanced. Similar observation was detected by Myrdal and Yalkowsky $^{16}$ who found that PEG 400 had a higher solubilizing action on phenytoin than PEG 200.

The solubilizing effect of glycerol, PG and PPG on the drug is shown in Figure 3 in which, PPG exhibited a higher solubilizing action than both PG and glycerol. These results agree with those obtained by Myrdal and Yalkowsky ${ }^{16}$ who found that PG exerted a higher solubilizing effect on benzocaine more than that obtained with glycerol. Furthermore, the solubilization of different pharmaceutical solutes by different cosolvents was studied and the results showed that PG was more efficient as a solubilizer than glycerol. ${ }^{17}$ This observation was attributed to a fact that solubilization decreased as the polarity of the solvent increased. The enhancement of tenoxicam aqueous solubility in presence of these hydroxylated co-solvents can be interpreted on a fact that: specific co-solvent solute interactions may occur which depend on the molecular structure of the solute and the cosolvent. These molecular interactions may result in the formation of hydrogen-bonded watersoluble complex. ${ }^{18}$

\section{Effect of different non-ionic surfactants on tenoxicam aqueous solubility}

Different classes of water-soluble non-ionic surfactants were studied for their solubilizing effects on tenoxicam. These include polysorbates (Tweens), polyoxyethylene alkylethers (Brijs), and polyoxyethylene stearates (Myrjs).

The solubilization of tenoxicam in different non-ionic surfactant solutions at $37^{\circ}$ is shown in
Figures 4-6. The tested non-ionic surfactants were used above their respective critical micelle concentrations (CMC). The solubility of the drug in the non-ionic surfactant solutions was increased linearly by increasing the surfactant concentrations.

The solubilization of a slightly watersoluble compound can be treated in terms of association equilibrium between the solutes and the micelles in a micellar solution. ${ }^{19}$ Thus:

$$
\mathrm{D}_{\mathrm{w}}+\mathrm{M} \stackrel{\mathrm{K}_{\mathrm{m}}}{\longleftrightarrow} \mathrm{D}_{\mathrm{m}}
$$

Where $D_{m}$ and $D_{w}$ are respectively, the drug solubilized in the micellar and bulk water, $M$ is the micellar concentration and $K_{m}$ is the distribution coefficient of the drug between the micellar and the bulk aqueous phases.

Accordingly, the following relationships were derived:

$$
\mathrm{K}_{\mathrm{m}}=\frac{\mathrm{S}_{\mathrm{m}}}{\mathrm{S}_{\mathrm{w}} \mathrm{M}} \ldots \ldots \ldots \ldots \ldots \ldots \ldots
$$

$$
\text { and } S_{t}=S_{m}+S_{w}=K_{m} S_{w} M+S_{w} \ldots
$$

Where $S_{m}$ and $S_{w}$ are, respectively, the solubility of the drug in the micellar and bulk aqueous phases, $M$ is the molar concentration of the micelle and $S_{t}$ is the total molar solubility of the drug in the micellar solution. Therefore, a plot of $S_{\mathfrak{l}}$ versus $M$ will produce a straight line with an intercept of $S_{w}$ and a slope of $K_{m} S_{w}$, which is the solubilizing capacity of the micelles. The slopes were determined by linear regression analysis. The distribution coefficient $\left(\mathrm{K}_{\mathrm{m}}\right)$ can be calculated from the slope $\left(K_{m} S_{w}\right)$ and $S_{w}$.

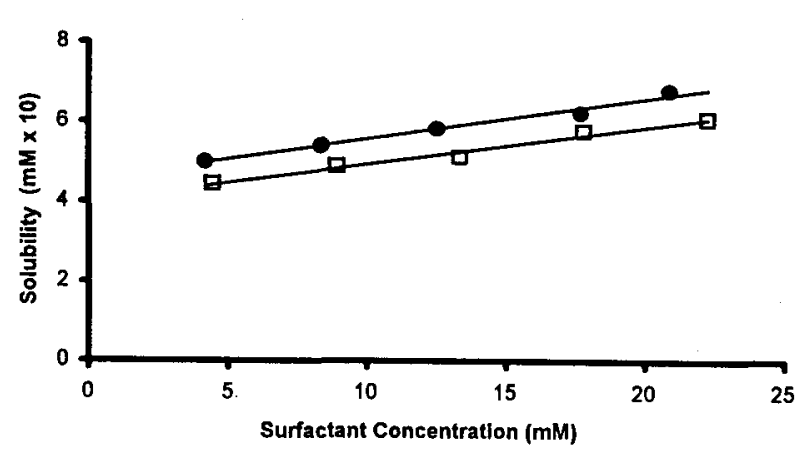

Fig. 4: Solubility of Tenoxicam in Aqueous Micellar Solution of Brij 35 and Brij 58 at $37^{\circ}$. Key: $\bullet$, Brij 35 and $\square$, Brij 58 . 


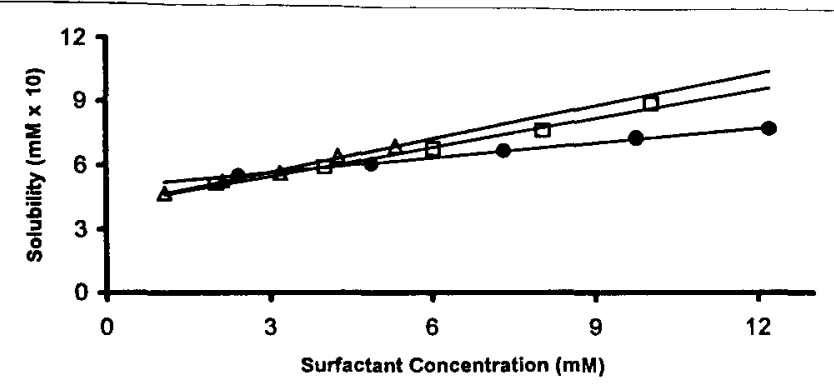

Fig. 5: Solubility of Tenoxicam in Aqueous Micellar Solution of Myrjs at $37^{\circ}$. Key: •, Myrj 52;, , Myrj 53 and $\Delta$, Myrj 59.

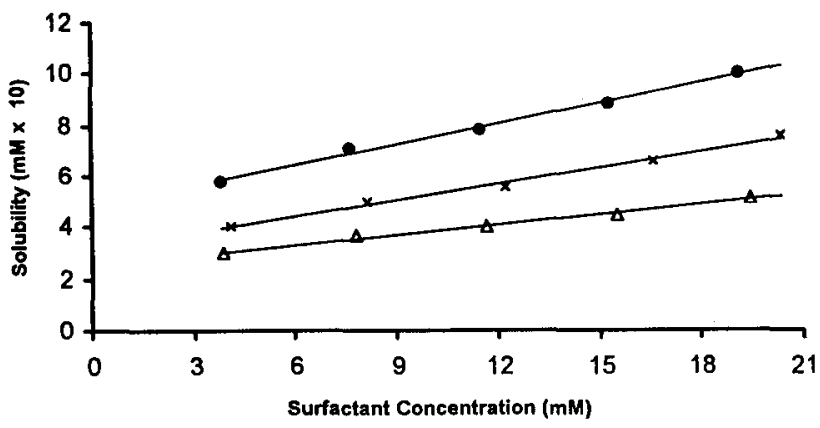

Fig. 6: Solubility of Tenoxicam in Aqueous Micellar Solution of Tweens at $37^{\circ}$. Key:

$\bullet$, Tween 80; $x$, Tween 60 and $\Delta$, Tween 40.

Table 1 lists the solubilizing capacity of the tested surfactants and the distribution coefficient of tenoxicam between micellar and bulk aqueous phases.

The solubilization of tenoxicam in solutions of two different polyoxyethylene alkylethers, i.e., Brijs, it was found that Brij 35 was more efficient as a solubilizer than Brij 58 as shown in Figure 4. In addition, it was found that Brij 35 exhibited a higher solubilizing capacity on tenoxicam than Brij 58 and the drug exhibited a higher distribution coefficient between Brij 35 micelles than Brij 58. This result agrees with the data obtained by Aboutaleb et $a l^{20}$ when levomepromazine and quinethazone were solubilized in solutions of Brij 35 and Brij 58 in which Brij 35 was more effective than Brij 58 as solubilizer. Furthermore, when $\mathrm{N}$-desmethyldiazepam was solubilized in solutions of Brij 35 and Brij 58, Brij 35 was found to be more efficient as a solubilizer than Brij 58..$^{21}$ Also, Samaha and Gadalla $^{22}$ studied the effect of Brij 35 and Brij 98 on the solubility of carbamazepine. The results showed that Brij 35 exhibited a better solubilizing action than Brij 98. They concluded that the change in the solubilization capacity between different Brijs is due to the difference between their hydrocarbon chain lengths.

The solubility data of tenoxicam in solutions of polyoxyethylene stearates with different hydrophilic chain lengths, i.e., Myrj 52, 53 and 59 are shown in Table 1 and Figure 5. On extending the polyoxyethylene chain, a pronounced increase in the solubility of the drug was observed. Therefore, Myrj 59 is more efficient than Myrj 53 and Myrj 52 as solubilizers. In addition, the effect of the polyoxyethylene stearates (Myrjs) on the solubility and distribution coefficient of tenoxicam can be ranked in a descending order as Myrj $59>$ Myrj 53>Myrj 52. The results obtained can be explained on the basis that, as the distribution coefficient of the drug between the micelles of Myrj increased, its aqueous solubility increased. Thus, the distribution coefficient of tenoxicam between the micelles of Myrjs can be considered as the main factor controlling micellar solubilization of the drug by Myrj series.

Polysorbates (Tweens) are non-ionic surfactants having the same hydrophilic portions in their molecules, but differ in their hydrophobic moiety. It is clearly evident that the solubilizing effect of these micellar-forming agents increases as the hydrophobic chain length of the surfactant increases. This may be due to the increase in the micellar core volume, which accommodate more quantities of such non-polar solute. ${ }^{23}$ Therefore, the micellar core volume can be considered as the main factor, which affects the solubilization of tenoxicam in Tweens. Accordingly, Tween 80 exhibited a better solubilizing capacity on tenoxicam than other investigated Tweens. On the other hand, the tested polysorbates (Tweens) could be arranged according to their solubilizing capacity on tenoxicam and the distribution coefficient of the drug between their micelles as Tween $80>$ Tween $60>$ Tween 40 . Raunio and Turakka ${ }^{24}$ studied the solubility of tolfenamic acid in 
Table 1: Solubilizing capacity of different surfactants and the distribution coefficient of tenoxicam between the micellar and aqueous phases.

\begin{tabular}{|l|c|c|}
\hline \multicolumn{1}{|c|}{ Surfactant } & $\begin{array}{c}\text { Solubilizing Capacity } \\
\times 10^{3}\end{array}$ & $\begin{array}{c}\text { Distribution Coefficient } \\
\left(\mathrm{mM}^{-1}\right) 10^{3}\end{array}$ \\
\hline Polyoxyethylene alkylethers (Brijs): & & \\
Brij 35 & 10.6 & 23.3 \\
Brij 58 & 9.4 & 22.4 \\
\hline Polyoxyethylene stearates (Myrjs): & & 44.3 \\
Myrj 52 & 22.1 & 107.6 \\
Myrj 53 & 44.6 & 123.0 \\
Myrj 59 & 50.9 & \\
\hline Polysorbates (Tweens): & & 46.1 \\
Tween 40 & 12.9 & 50.9 \\
Tween 60 & 20.1 & 55.0 \\
Tween 80 & 26.7 & \\
\hline
\end{tabular}

solutions of different tweens. They noted that Tween $80>$ Tween $60>$ Tween 40 as solubilizers and they attributed this finding to the fact that the solubilizing capacity of the surfactants increases linearly with increasing relative amount of the hydrophobic part. Also, similar observation was detected when some anti-inflammatory drugs were solubilized via different Tweens. The results revealed that, Tween $80>$ Tween $60>$ Tween 40 as solubilizers. ${ }^{25}$

\section{Characterization of tenoxicam solid dispersions with different polymers \\ Dissolution rate studies}

Figure 7 shows the dissolution profiles of tenoxicam-PEG4000 solid dispersion systems in different drug: polymer ratios $(4: 1,3: 2,2: 3,1: 4$, and 1:9). Preparations containing 9 parts of PEG 4000 display $D_{60}$ values of $0.246 \mathrm{mg} / \mathrm{ml}$ $\left[D_{60}\right.$ is the cumulative amount $(\mathrm{mg} / \mathrm{ml})$ of tenoxicam dissolved after 60 minutes].

A comparison of the dispersions containing different drug: polymer ratios with PEG 4000 shows that the higher the amount of PEG, the higher the dissolution rate of the medicament. These data agree with those obtained by Doshi et $a l^{26}$ who showed that the dissolution rate of carbamazepine from its solid dispersions with PEG 4000 and PEG 6000 increased greatly by increasing the polymeric ratio. In addition, Kassem et $a l^{27}$ studied the effect of the polymeric ratio on the dissolution of chloramphenicol from its solid dispersions with PEG 4000 and PEG 6000 . They observed that the dissolution rate was increased by increasing the polymer ratio and a higher dissolution rate was exhibited using drug: polymer ratio of (1:9).

The dissolution profile of tenoxicam from its solid dispersion with PEG 4000 in drug: polymer ratio of (1:9) and its corresponding physical mixture was compared and the data were displayed in Figure 8. The physical mixture does not exhibit a significant increase in the dissolution rate of drug than the untreated drug. However, solid dispersions prepared using PEG 4000 displayed faster and higher dissolution rates than the corresponding physical mixture.

The plots of the cumulative amount of the drug dissolved from tenoxicam-PVP 40000 solid dispersions in different ratios are shown in Figure 9. The results obtained demonstrated that the dissolution rate of tenoxicam was markedly increased by increasing the ratio of the carrier in the tested system, being maximum at a drug: polymer ratio of (1:9). The $D_{60}$ values of tenoxicam in solid dispersion with PVP $40000 \mathrm{was} .0 .323 \mathrm{mg} / \mathrm{ml}$. The ratio (1:9) of tenoxicam-PVP solid dispersion system exhibited faster dissolution rate of the medicament than the corresponding physical mixture as well as the untreated drug (Figure 10). The improvement of the dissolution rate of 


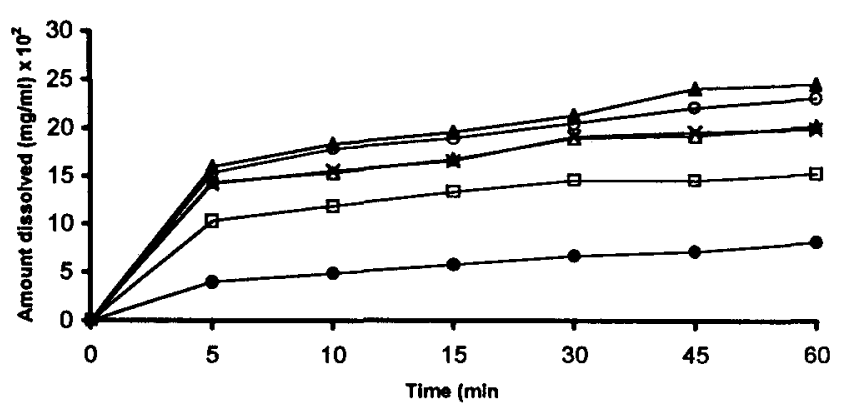

Fig. 7: Dissolution Rate Profile of Tenoxicam from its- PEG 4000 Co-precipitates at $37^{\circ}$. Key: •, Untreated Drug; $\square, 4: 1 ; \Delta, 3: 2 ; x$, $2: 3 ; 0,1: 4$ and $\Delta, 1: 9$.

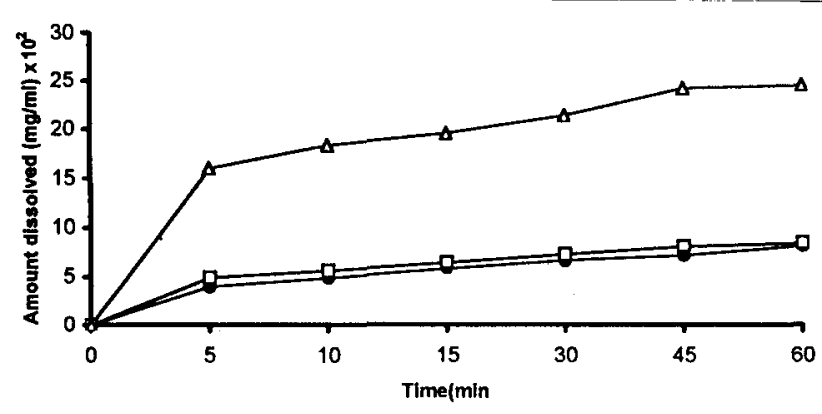

Fig. 8: Dissolution Rate Profile of Tenoxicam from its Co-precipitate and Physical Mixture with PEG 4000 in (1:9) Ratio w/w at $37^{\circ}$. Key: •, Untreated Drug; $\square$, Physical Mixture and $\Delta$, Coprecipitate.

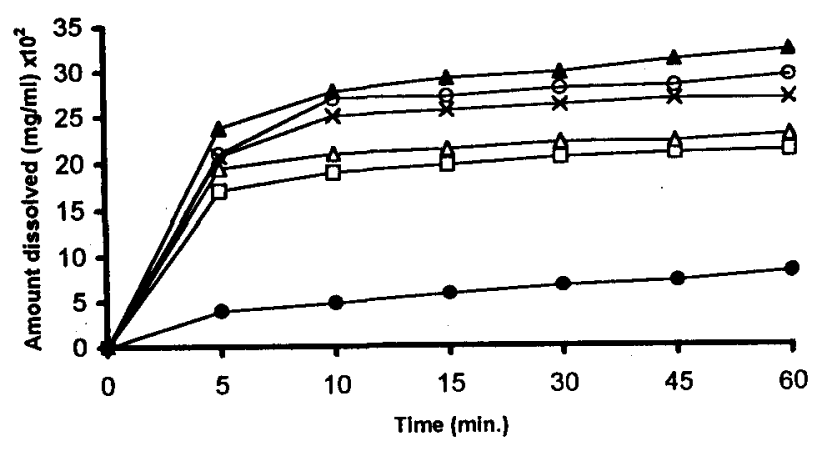

Fig. 9: Dissolution Rate Profile of Tenoxicam from its- PVP 40000 Co-precipitates at $37^{\circ}$. Key: •, Untreated Drug; $\square, 4: 1 ; \Delta$, $3: 2 ; x, 2: 3 ; 0,1: 4$ and $\Delta, 1: 9$.

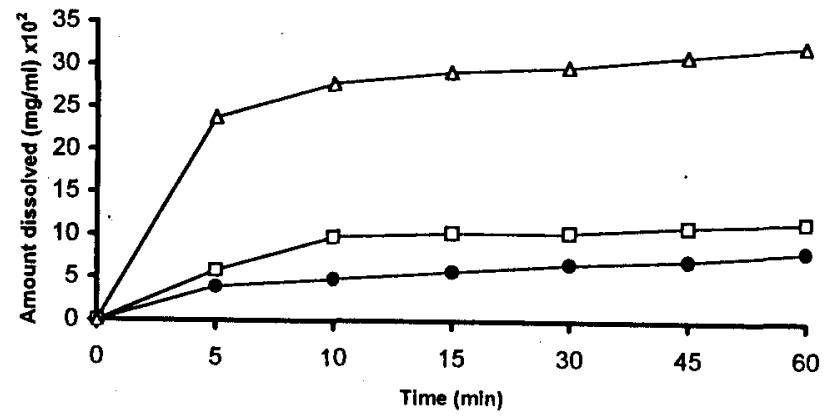

Fig. 10: Dissolution Rate Profile of Tenoxicam from its Co-precipitate and Physical Mixture with PVP 40000 in (1:9) w/w Ratio at $37^{\circ}$. Key: •, Untreated Drug; $\square$, Physical Mixture and $\Delta$, Coprecipitate.

the drug from its solid dispersions might be due to the conversion of its crystalline form into the amorphous one, the reduction of the drug particle size, or an improvement in its wettability caused by the hydrophilic polymers used. $^{28}$ The dissolution rate of tenoxicam from physical mixtures with PVPs was greater than that of the untreated drug. This may be explained in terms of the possible lowering of the surface tension of the medium by PVPs, resulting in better wetting of the drug crystal surface. This is in accordance with the findings of Sekikawa et al. ${ }^{29}$ who studied the dissolution behavior of phenytoin from its solid dispersions with different polyvinylpyrrolidones. They found that phenytoin in the physical mixture dissolved faster than phenytoin alone. They stated that PVP in the medium from the physical mixture might lower the surface tension of the medium, resulting in the wetting of the hydrophobic drug crystalline surface. ${ }^{29}$

From the previous discussion, it is revealed that tenoxicam in PVP 40000 dispersion exhibited faster dissolution rates compared to its dispersions with PEG 4000. This increase in the dissolution rate of tenoxicam may be attributed to the fact that tenoxicam in a solid dispersion system may simultaneously crystallize out in very minute crystals embodied in water-soluble matrices. This causes an increase in the specific surface area of the drug leading to an increase in its dissolution rate..$^{30-32}$ Additionally, the rapid 
dissolution of these water-soluble matrices is accompanied by rapid dissolution of embodied minute drug crystals. Polymer encircling the drug decreases aggregation and agglomeration of drug particles, which can readily dissolve the drug and cause water to contact and wet the drug particles and so increase its dissolution rate.

The aqueous solubility of tenoxicam in its PEG 4000 PVP 40000 co-precipitates (1:9) was estimated. The drug exhibited aqueous solubility of 1.96 and $0.18 \mathrm{mg} / \mathrm{ml}$ in its co-precipitates with PVP 40000 and PEG 4000, respectively. On the other hand, the corresponding physical mixtures with these polymers did not exhibit any considerable increase in the drug aqueous solubility.

It can be concluded that tenoxicam dissolution rate was markedly improved via solid dispersion technique with PVP 40000 compared to PEG 4000 system. This enhancement in the hydrophilic characters of the drug using solid dispersion technique may be due to the change in its physicochemical properties. Therefore, DSC, IR spectrophotometry and X-ray diffraction studies were conducted to illustrate the possible mechanism of the enhanced dissolution rates of the drug from its solid dispersions with PVP and PEG 4000 .

\section{IR spectroscopy}

In order to characterize possible interactions between the drug and the polymeric carriers in the solid state, infrared spectra were recorded.

Figures $11 \& 12$ demonstrate IR spectra of the untreated tenoxicam, the polymers (PEG 4000 and PVP 40000), solid dispersions of the drug with the two polymers (1:9 weight ratio of drug: polymer) and the corresponding physical mixtures.

The top spectrum of each Figure (A) refers to the IR absorption spectrum of the untreated drug and it was identical with the reported data. ${ }^{33}$ According to these data, in the $\mathrm{N}-\mathrm{H}$ and $\mathrm{O}-\mathrm{H}$ frequency region, tenoxicam showed a band at $3420 \mathrm{~cm}^{-1}$, which was due to the overlapped. $\mathrm{N}-\mathrm{H}$ and $\mathrm{O}-\mathrm{H}$ vibrations. In addition, a strong band was observed at 1627 $\mathrm{cm}^{-1}$ which was attributed to the amide carbonyl stretching band $(\mathrm{C}=0)$; (Table 2). These two bands will be infocussed in the present study in order to trace the possibility of interaction of tenoxicam with either PEG or PVP.

Table 2: IR spectra of tenoxicam-PEG 4000 and tenoxicam-PVP 40000 coprecipitates (1:9 weight ratio of the drug: polymer) as well as the corresponding physical mixtures compared with the individual components.

\begin{tabular}{|l|c|c|}
\hline \multirow{2}{*}{ System } & \multicolumn{2}{|c|}{$\Delta \lambda \lambda_{\max }\left(\mathrm{cm}^{-1}\right)$} \\
\cline { 2 - 3 } & $\begin{array}{c}\text { O-H and N-H } \\
\text { Stretching }\end{array}$ & $\begin{array}{c}\mathrm{C}=\mathrm{O} \\
\text { Stretching }\end{array}$ \\
\hline Untreated Tenoxicam & $\begin{array}{c}3420 \\
\text { (overlapped) }\end{array}$ & 1627 \\
\hline PEG 4000 & $\begin{array}{c}3445 \\
\text { (O-H Stretching) }\end{array}$ & - \\
\hline Tenoxicam- PEG 4000 Phys. Mix. & $\begin{array}{c}3515 \\
\text { (overlapped) }\end{array}$ & 1629 \\
\hline Tenoxicam- PEG 4000 Coppt. & $\begin{array}{c}3465 \\
\text { (overlapped) }\end{array}$ & 1634 \\
\hline PVP 40000 & - & 1633,1679 \\
\hline Tenoxicam- PVP 40000 Phys. Mix. & $\begin{array}{c}3475 \\
\text { (overlapped) }\end{array}$ & 1664 \\
\hline Tenoxicam- PVP 40000 Coppt. & 3430 & \\
\hline
\end{tabular}



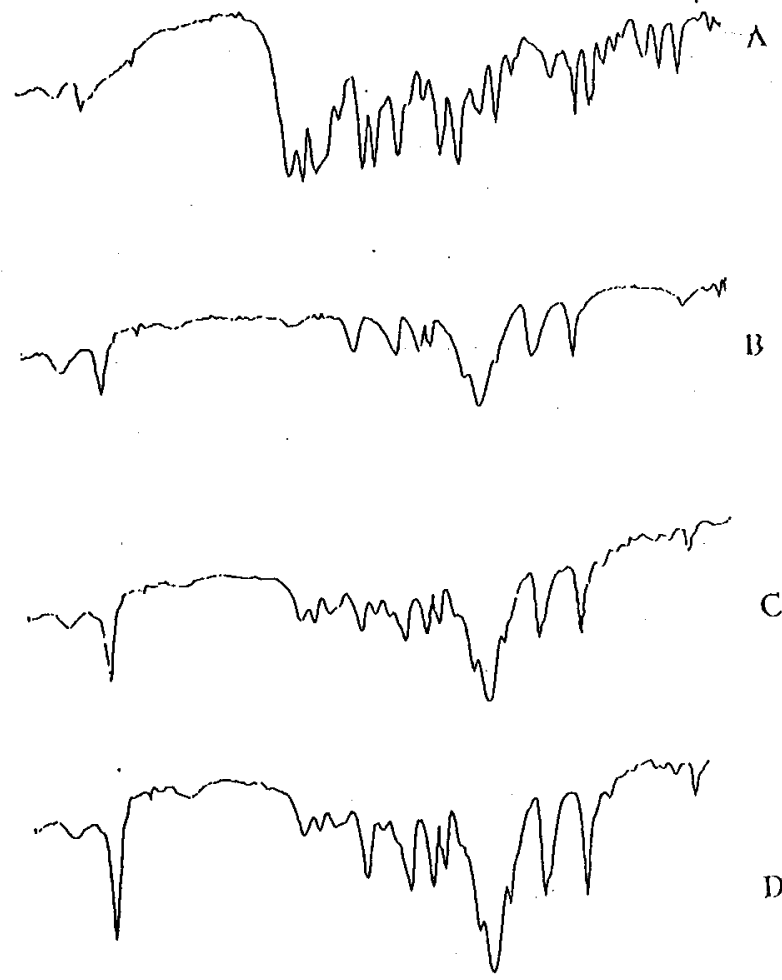

$40002000 \quad 1600 \quad 800$

Wave number cut

Fig. 11: The IR spectra of Tenoxicam coprecipitate with PEG 4000; A, Untreated Tenoxicam; B, PEG 4000 alone; C, Tenoxicam-PEG 4000 (1:9) Phyical Mixture and D, Tenoxicam-PEG 4000 (1:9) Coprecipitate weight ratios.

The IR absorption spectra for tenoxicamPEG 4000 solid dispersion (1:9) and the corresponding physical mixture as well as the individual components are shown in Figure 11 and Table 2. The band at $3420 \mathrm{~cm}^{-1}$ which refers to the overlapped $\mathrm{N}-\mathrm{H}$ and $\mathrm{O}-\mathrm{H}$ stretching vibrations of tenoxicam was shifted from 3420 $\mathrm{cm}^{-1}$ to $3515 \mathrm{~cm}^{-1}$ and $3465 \mathrm{~cm}^{-1}$ in case of tenoxicam-PEG 4000 physical mixture and coprecipitate, respectively. However, these shifts in the band position $\left(95 \mathrm{~cm}^{-1}\right.$ and $65 \mathrm{~cm}^{-1}$, respectively) were considered to be nonsignificant. ${ }^{34}$ Moreover, the carbonyl stretching band of the drug in case of physical mixture and solid dispersion with PEG 4000 was observed almost at its original position; $1629 \mathrm{~cm}^{-1}$ and $1634 \mathrm{~cm}^{-1}$, respectively.

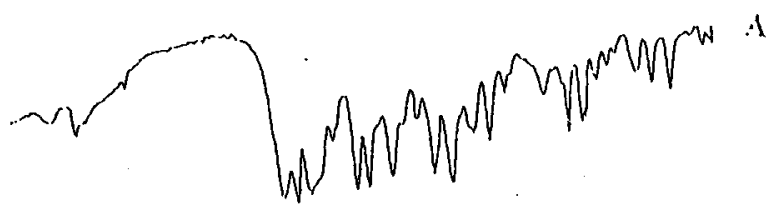

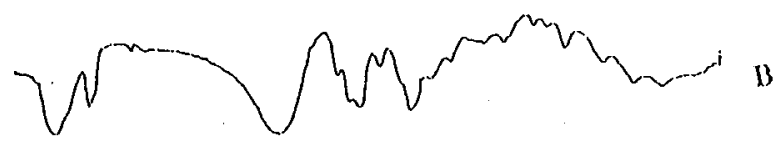

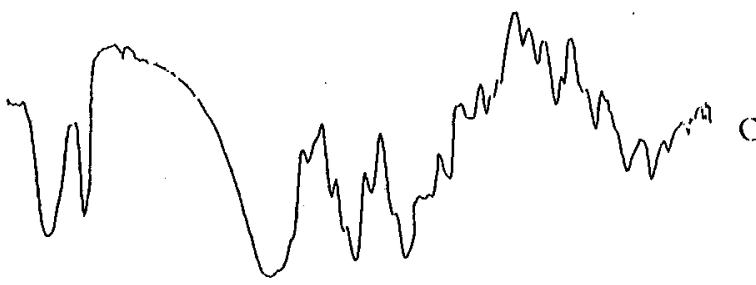

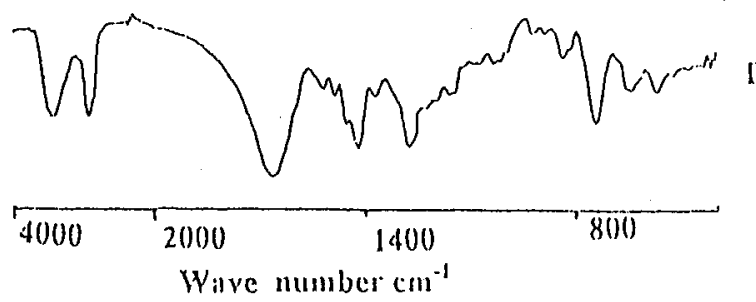

Fig. 12: The IR spectra of Tenoxicam coprecipitate with PVP 40000; A, Untreated Tenoxicam; B, PVP 40000 alone; C, Tenoxicam-PVP 40000 (1:9) Phyical Mixture and D, Tenoxicam-PVP 40000 (1:9) Coprecipitate weight ratios.

The IR spectra of tenoxicam solid dispersion with PVP 40000 (1:9) and their physical mixture were recorded. The spectra of the tested PVP (curve B in Figure 12) showed $\mathrm{C}=\mathrm{O}$ stretching band at $1668 \mathrm{~cm}^{-1}$, Table 2 . The overlapped $\mathrm{N}-\mathrm{H}$ and $\mathrm{O}-\mathrm{H}$ stretching vibrations $\left(3420 \mathrm{~cm}^{-1}\right)$ for tenoxicam did not show any significant change in the physical mixture of the drug with PVP 40000 and were also seen overlapped at $3475 \mathrm{~cm}^{-1}$. In addition, this mixture of tenoxicam with PVP 40000 showed the broad bands at $1633 \mathrm{~cm}^{-1}$ and $1679 \mathrm{~cm}^{-1}$ which were the superimposed peaks between tenoxicam and the polymer (spectrum C). On the other hand, the IR spectrum of tenoxicamPVP 40000 solid dispersion (spectrum D) exhibited apparent changes specially in the 
carbonyl stretching region in which one band only appeared at $1664 \mathrm{~cm}^{-1}$. From above mentioned results, we might suggest that there is some sort of interaction between tenoxicam and the tested PVP. This interaction may be due to the presence of $\mathrm{C}=\mathrm{O}$ group in the PVP molecules (electron donor) and both $\mathrm{O}-\mathrm{H}$ and $\mathrm{N}$ $\mathrm{H}$ group in the studied drug (electron acceptor), the possibility of hydrogen bonding is predominant. The $\mathrm{O}-\mathrm{H}$ group of the drug may be largely involved in an intramolecular hydrogen bonding with the $\mathrm{C}=\mathrm{O}$ group in the same molecule (the intramolecular hydrogen bonding is more stable than the intermolecular hydrogen bonding). Thus, the probability of the interaction of the $\mathrm{N}-\mathrm{H}$ group of the drug with the $\mathbf{C}=\mathbf{O}$ of PVP is higher. As reported in the literature. ${ }^{34}$ hydrogen bonding causes a shift in the $\mathrm{X}-\mathrm{H}(\mathrm{N}-\mathrm{H}$ or $\mathrm{O}-\mathrm{H})$ group from about 100 to $300 \mathrm{~cm}^{-1}$ and about $15 \mathrm{~cm}^{-1}$ for $\mathrm{C}=0$ group. From Figure 12 and Table 2, it is obvious that there are considerable shifts $\left(15 \mathrm{~cm}^{-1}\right)$ in the $\mathrm{C}=\mathrm{O}$ positions of PVP from $1679 \mathrm{~cm}^{-1}$ to 1664 $\mathrm{cm}^{-1}$ and this agrees well with the reported data. $^{34}$ Unfortunately, the shift in the X-H $(\mathrm{N}-\mathrm{H}$ or $\mathrm{O}-\mathrm{H}$ ) band of tenoxicam is not detected due to the interference from the very strong broad band of PVP around $3430 \mathrm{~cm}^{-1}$ (due to presence of moisture in the PVP sample). These findings agree with Tantishaiyakul et al. ${ }^{35}$ who noticed that both $\mathrm{N}-\mathrm{H}$ and $\mathrm{O}-\mathrm{H}$ stretching bands of piroxicam were shifted toward lower wavenumber in case of solid dispersion with PVP. They attributed this observation to the presence of the intermolecular hydrogen bonding between $\mathrm{N}-\mathrm{H}$ and $\mathrm{O}-\mathrm{H}$ stretching of piroxicam and $\mathrm{C}=\mathrm{O}$ of PVP $\mathrm{K}$ during the dispersion process. Furthermore, Hamza et al. ${ }^{36}$ studied the interaction between indometacin with PVP in the solid dispersion form. They observed that both the carboxylic $\mathrm{O}-\mathrm{H}$ deformation and $\mathrm{C}-\mathrm{H}$ deformation bands were shifted to higher frequencies in the solid dispersion form may be due to the drug-polymer interaction via the $\mathrm{C}=0$ group. Van den Mooter et al. ${ }^{37}$ suggested that when interaction is expected between temazepam and PVP in the solid state, it should involve the $\mathrm{O}-\mathrm{H}$ group of temazepam and the carbonyl group of the polymer in hydrogen bonding.

Thus, from the IR spectral analysis, it might be suggested that there was no physicochemical interaction between tenoxicam and PEG4000. However, this analysis confirmed the interaction between the drug and PVP 40000.

\section{Differential Scanning Calorimetry (DSC):}

In order to shed a light on the possibility of solid state changes of tenoxicam with either PEG 4000 or PVP 40000 , DSC was performed on drug-carrier coprecipitates (1:9 weight ratio of drug: polymer) and their physical mixtures as well as the individual components. The DSC curves of untreated tenoxicam (Figures $13 \&$ 14 , curve A) show an endothermic peak at $218.5^{\circ}$ with a thaw point at $208^{\circ}$ and $\Delta \mathrm{H}-115.6$ joule/g at a scanning rate of $10 \% \mathrm{~min}$. This endothermic peak ends with an exothermic peak at $223^{\circ}$, which may be due to the decomposition of the drug when reaching its melting point as reported. $^{33}$

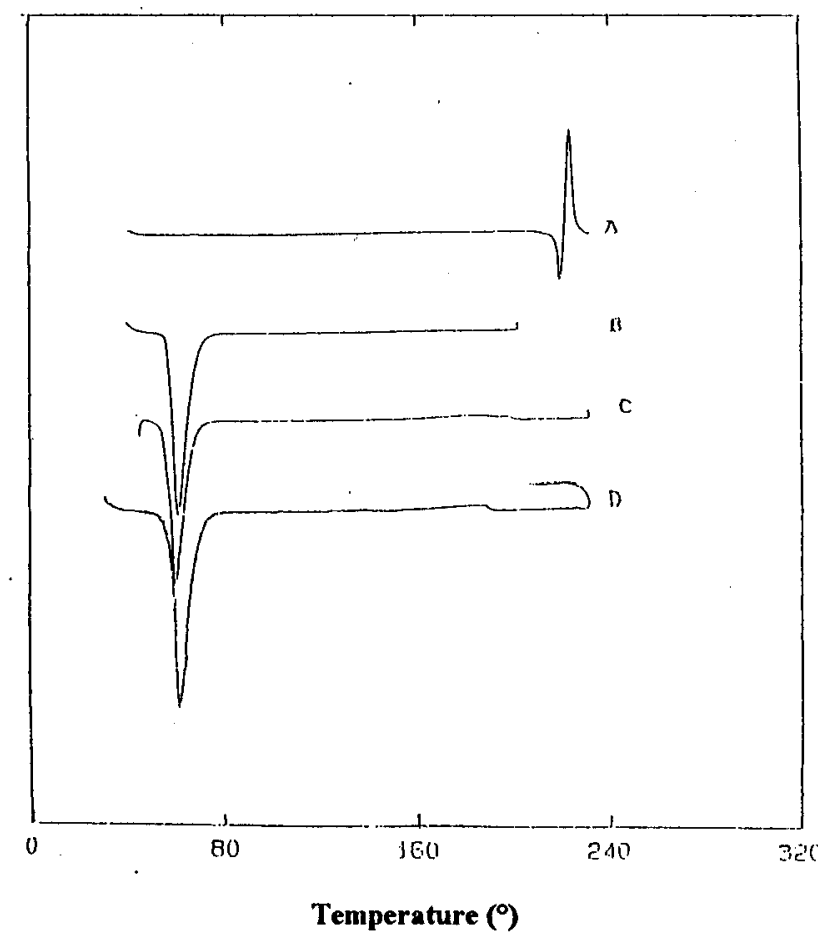

Fig. 13: DSC curves of Tenoxicam Coprecipitae with PEG 4000 at Scanning Speed of $10 \%$ min.; A, Untreated Tenoxicam; B, PEG 4000 alone; C, Tenoxicam-PEG 4000 (1:9) Physical Mixture and D, TenoxicamPEG 4000 (1:9) Coprecipitate Weight Ratios. 


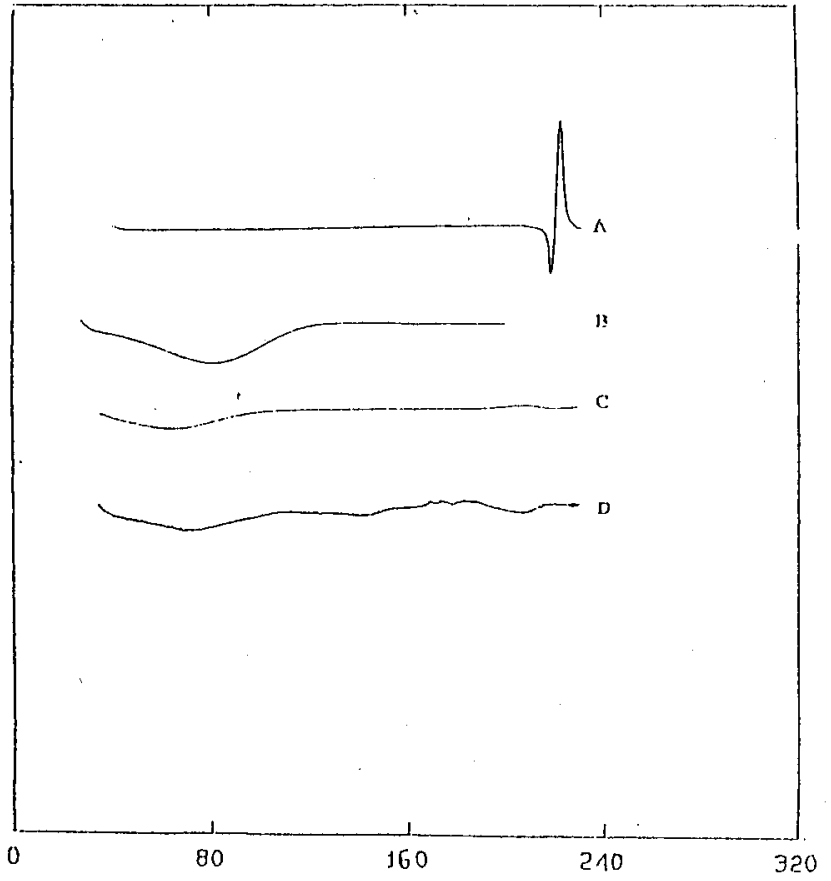

Temperature $\left({ }^{\circ}\right)$

Fig. 14: DSC curves of Tenoxicam Coprecipitae with PVP 40000 at Scanning Speed of $10 \%$ min.; A, Untreated Tenoxicam; B, PVP 40000 alone; C, Tenoxicam-PVP 40000 (1:9) Physical Mixture and D, Tenoxicam-PVP 40000 (1:9) Coprecipitate Weight Ratios.

The DSC curves of tenoxicam, PEG 4000, 1:9 w/w tenoxicam-PEG 4000 solid dispersion and their physical mixture are shown in Figure 13. This figure displays the endothermic peak of PEG 4000 at $59.8^{\circ}$ (curve B) and disappearance of that of tenoxicam in both physical mixture and solid dispersion. In addition, the drug exothermic peak disappeared in both cases.

DSC tracing of tenoxicam-PVP 40000 (1:9) solid dispersion, their physical mixture and the individual components are depicted in Figure 14. It was clear that the PVP plot (curve B) showed a shallow, broad endothermic peak at about $81^{\circ}$ which was thought to represent the vaporization of moisture from the PVP sample which confirmed the previous observation found in the IR spectra of this polymer. DSC thermogram of tenoxicam-PVP 40000 physical mixture showed that the endothermic peak of the drug exists at the same position compared to the untreated drug $\left(219.6^{\circ}\right.$ with a thaw point $211.9^{\circ}$ ) but it lost its distinct sharpened appearance. On the other hand, this characteristic endothermic peak was shifted to a lower temperature $\left(206^{\circ}\right.$ with a thaw point $186.9^{\circ}$ ) compared to the untreated drug on scanning the drug-PVP 40000 solid dispersion as shown in Figure 14: D. Furthermore, the exothermic peak of the drug completely disappeared in case of tenoxicam solid dispersion and physical mixture with this polymer. In addition, a reduction was observed in the $\Delta \mathrm{H}$ value of tenoxicam from a value of 115.6 to -20.77 and -4.66 joule/g in case of solid dispersion and physical mixture, respectively.

As mentioned above, the characteristic endothermic peak of tenoxicam in its-polymer solid dispersions almost disappeared or reduced in intensity, shifted to lower temperatures and lost its sharpened distinct appearance. Also, the drug exhibited lower values of $\Delta \mathrm{H}$ in the dispersions with the tested polymers. This observation confirms the presence of tenoxicam in an amorphous form in these solid dispersions. Similar DSC results were obtained by Najib and Suleiman $^{38}$ who noticed the disappearance of the endothermic peak of PEG 4000 solid dispersions with diflunisal. They explained this phenomenon on basis that solid dispersion resulted in an amorphous form of the drug. In addition, AlAngary et al. ${ }^{39}$ showed that lorazepam endothermic peak disappeared in its-PEG 10000 solid dispersions. They stated that this phenomenon can be correlated to an amorphous state of the drug present in the solid dispersion. Other investigators supported this explanation. ${ }^{40.41}$ However, the disappearance of the endothermic peak corresponds to the melting of tenoxicam in the drug-polymer physical mixture may be due to its solubility in the melted polymer. This finding agrees with Simonelli et al. ${ }^{42}$ who showed the disappearance of the endothermic peak of hydrochlorothiazide in both physical mixture and solid dispersion with PEG 6000, which might indicate the solubility of the drug in the molten polymer during running the thermograms. Moreover, Khidr $^{43}$ found the same behavior when he studied the solid dispersion of nifedipine with pluronic F-127. 


\section{X-ray diffractometry}

To get further evidence on the solid state changes, $x$-ray diffraction spectra were carried out on tenoxicam, tenoxicam-PVP 40000 coprecipitate (1:9 weight ratio of drugs: polymer) and their physical mixtures as well as the individual components.

The presence of numerous distinct peaks in the $x$-ray diffraction spectrum of tenoxicam indicates that tenoxicam is present as a crystalline material with characteristic diffraction peaks appearing at diffraction angels of $2 \theta$ at $7.58 \mathrm{~A}^{\circ}, 3.80 \mathrm{~A}^{\circ}, 3.44 \mathrm{~A}^{\circ}, 3.39 \mathrm{~A}^{\circ}$ and $3.03 \mathrm{~A}^{\circ}$ with relative intensities of $43.45,100$, $56.97,41.47$ and 54.99 , respectively (Figure 15: $A$ and Table 3). However, the spectrum of the tested PVP was characterized by the complete absence of any diffraction peak (Figure 15: B).

The spectrum of tenoxicam-PVP 40000 (1:9) solid dispersion (Figure 15: D and Table 3) shows a reduction of the intensity of the drug characteristic peaks $(9.32,21.02,21.52,18.38$, 18.96 respectively). On the other hand, in the physical mixture of the same composition (Figure 15: $\mathrm{C}$ and Table 3), these characteristic peaks are smaller than those of the untreated drug but still show a higher diffraction intensity compared to the solid dispersion system (33.39, $52.35,45.10,41.8$, and 42.62 , respectively).

The data obtained from the $x$-ray diffraction studies on tenoxicam-PVP (1:9) coprecipitate indicated that PVP might inhibited the crystallization of tenoxicam and resulted in an amorphous form of the drug. These results are in agreement with several authors ${ }^{44.48}$ who found that drugs-PVP coprecipitates showed disappearance of the $\mathrm{x}$-ray diffraction peaks of the drug crystals.

The effect of aging on the physicochemical properties of tenoxicam-PVP 40000 (1:9) coprecipitate was carried out by storing the formulation in a desiccator over calcium chloride for 9 months and examining the $x$-ray diffraction behavior of this coprecipitate. The results revealed that the storage of the coprecipitate did not affect its $x$-ray diffraction pattern indicating that tenoxicam still present as an amorphous form in its-PVP 40000 coprecipitate.
As mentioned previously, the dissolution rate of tenoxicam has been improved by solid dispersion of the drug with PVP. Thus, the amorphous state of the drug might play a fundamental role in this improvement via solid dispersion technique.

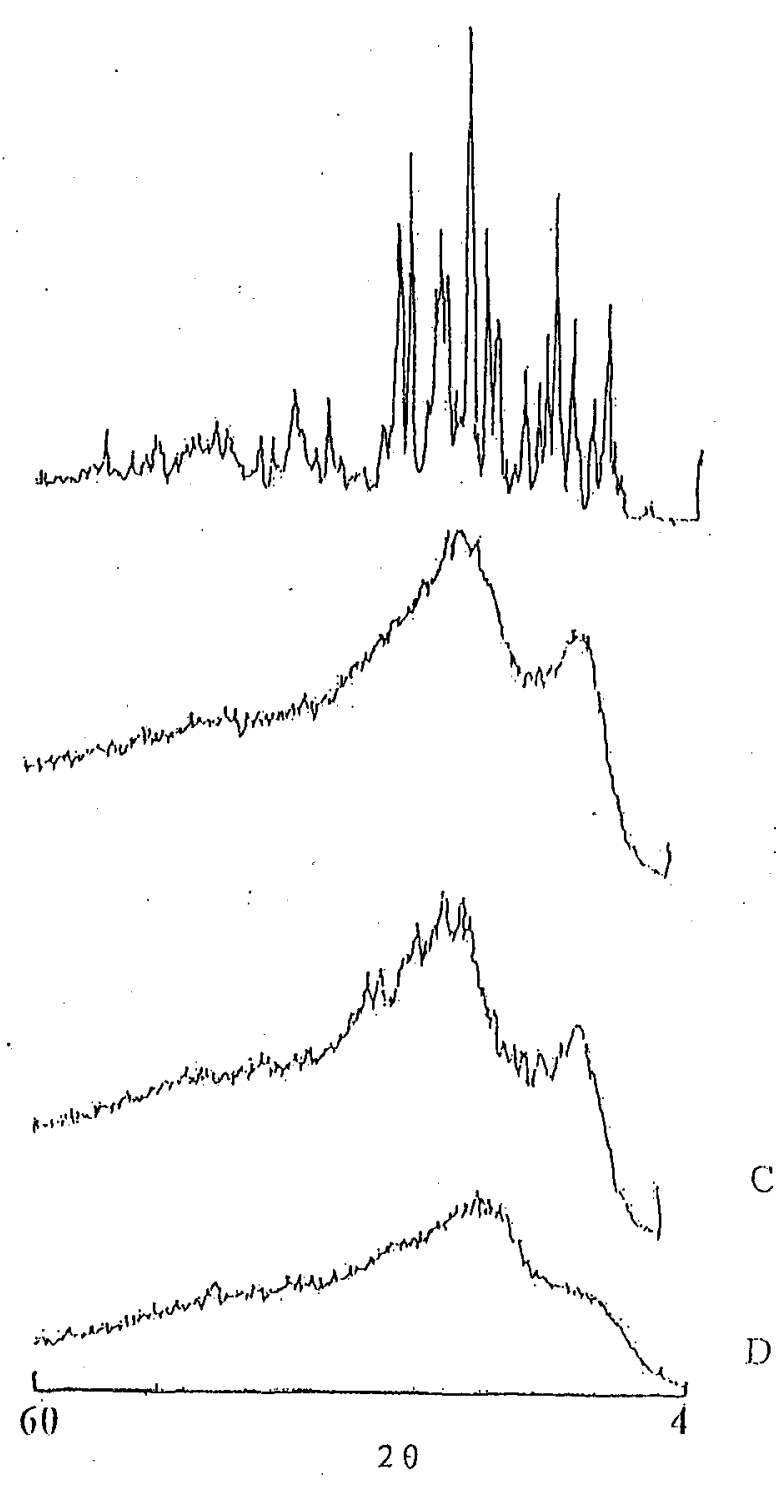

Fig. 15: X-ray Powder Diffraction Patterns of Tenoxicam Coprecipitate with PVP 40000: A, Untreated Tenoxicam; B, PVP 40000 alone; C, Tenoxicam-PVP 4000 (1:9) Physical Mixture and D, Tenoxicam-PVP 40000 (1:9) Coprecipitate Weight Ratios. 
Table 3: X-ray diffraction of tenoxicam-PVP 40000 coprecipitate (1:9 weight ratio of drug: polymer) as well as the corresponding physical mixture compared with the untreated drug expressed by the relative diffraction intensity.

\begin{tabular}{|c|c|c|c|c|}
\hline \multirow{3}{*}{$2 \theta \theta \theta$} & \multirow{3}{*}{$\mathrm{D}\left(\mathrm{A}^{\circ}\right)$} & \multicolumn{3}{|c|}{ Relative Diffraction Intensities of Tenoxicam in Presence of: } \\
\hline & & \multirow{2}{*}{$\begin{array}{l}\text { Untreated } \\
\text { Drug }\end{array}$} & \multicolumn{2}{|c|}{ PVP 4000} \\
\hline & & & $\mathrm{Ph} . \mathrm{M}$ & Coppt. \\
\hline 11.682 & 7.5749 & 43.45 & 33.39 & 9.32 \\
\hline 12.857 & 6.8852 & 26.71 & 32.89 & 12.28 \\
\hline 14.654 & 6.0446 & 42.132 & 30.09 & 14.35 \\
\hline 17.556 & 5.0516 & 27.95 & 30.59 & 15.25 \\
\hline 18.641 & 4.7600 & 28.52 & 35.37 & 18.55 \\
\hline 21.065 & 4.2174 & 37.02 & 49.05 & 22.84 \\
\hline 23.450 & 3.7935 & 100 & 52.35 & 21.02 \\
\hline 25.916 & 3.4379 & 56.97 & 45.10 & 21.52 \\
\hline 26.330 & 3.3848 & 41.47 & 41.80 & 18.38 \\
\hline 28.493 & 3.3126 & 70.98 & 44.52 & 18.55 \\
\hline 29.464 & 3.0315 & 54.99 & 42.62 & 18.96 \\
\hline 35.443 & 2.5326 & 23.50 & 28.28 & 13.85 \\
\hline
\end{tabular}

\section{Conclusions}

From the previous study, it could be concluded that:

1- Tenoxicam aqueous solubility has been improved using different additives. Formamide and dimethyl formamide exhibited the highest solubilizing power on tenoxicam, while hydroxylated co-solvents just potentiated its aqueous solubility.

2- Non-ionic surfactants caused a pronounced enhancement in the aqueous solubility of tenoxicam. Tween 80 showed the highest solubilizing action on tenoxicam than the other tested non-ionic surfactants.

3- Solid dispersion of tenoxicam in PEG 4000 and PVP 40000 improved both dissolution rate as well as aqueous solubility of the drug especially PVP 40000 in a drug: polymer ratio of (1:9). The aqueous solubility of tenoxicam was enhanced about 22 times in its-PVP 40000 solid dispersion (1:9).
The mechanisms responsible for this enhancement in the hydrophilic characteristics of drug via solid dispersion technique can be summarized as follows:

a) The presence of tenoxicam in an amorphous form in its dispersions with PVP as proved by $x$-ray and DSC analysis.

b) An interaction between the drug and PVP by hydrogen bonding of the drug with pyrrolidone moiety in the solid dispersion form has been occurred as elucidated from IR studies.

\section{REFERENCES}

1- A. M. Shawesh, S. Kallioinen, L. Hellen and J. Yliruusi, Pharmazie, 53, 567 (1998).

2- W. Bialik, K. A. Walters, K. R. Brain and J. Hadgraft, Int. J. Pharm., 92, 219 (1993).

3- K. Sugibayashi, M. Nemoto and $Y$. Morimoto, Chem. Pharm. Bull., 36,1519 (1988).

4- A. H. Ghanem, H. El-Sabbagh and $\mathrm{H}$. Abdel-Alim, Pharm. Ind., 42, 854 (1980). 
5- H. O. Ammar and S. A. El-Nahhas, Pharmazie, 49, 583 (1994).

6- A. A. Ismail and M. A. S. Gadalla, Ibid, 38, 733 (1983).

7- M. A. A. Kassem, G. Elmahrouk and A. A. Kassem, Bull. Pharm. Sci., Assiut University, 6, 361 (1984).

8- K. M. S. Fahelelbom, R. F. Timoney and O. I. Corrigan, Pharm. Res., 10,631 (1993).

9- K. Sekiguchi and N. Obi, Chem. Pharm. Bull., 9, 861 (1961).

10- W. R. Ravis and C. Chen, J. Pharm. Sci., 70, 1353 (1983).

11- A. M. Rabasco, J. M. Gines, M. Fernandez-Arevalo and M. A. Holgado, Int. J. Pharm., 67, 201 (1991).

12- M. T. Shen, C. M. Yeh and T. D. Sokoloski, Ibid, 103, 137 (1994).

13- G. V. Betageri and K. R. Makarla, Ibid, 126,155 (1995).

14- W. L. Chiou and S. R. Riegelman, J. Pharm. Sci., 60, 281 (1971).

15- S. A. El-Harras, Bull. Pharm. Sci., Assiut University. 20, 95 (1997).

16- P. B. Myrdal and S. H. Yalkowsky, In: "Encyclopedia of Pharmaceutical Technology", J. Swarbrick and J. C. Boylan (Eds.). Dekker, New York, 18 (1999), p. 185.

17- S. H. Yalkowsky and T. J. Roseman, In: "Techniques of Solubilization of Drugs", S. H. Yalkowsky (Ed.), Dekker, New York, (1985), p. 93-119.

18- P. B. Myrdal and S. H. Yalkowsky, In: "Encyclopedia of Pharmaceutical Technology", J. Swarbrick and J. C. Boylan (Eds.). Dekker, New York, 18 (1999), p. 187.

19- J. T. H. Ong and E. Manoukian, Pharm. Res., 5, 704 (1988).

20- A. E. Aboutaleb, A. A. Ali and R. B. Salama, Pharmazie, 36, 35 (1981).

21- A. E. Aboutaleb, A. A. Abdel Rahman and E. M. Samy, Bull. Pharm. Sci., Assiut University. 9, 119 (1986).

22- M. W. Samaha and M. A. F. Gadalla, Drug Dev. Ind. Pharm., 13, 93 (1987).

23- K. Sivakumar and M. B. Mithal, Ind. J. Pharm. Sci., Sept-Osct., 157 (1978).

24- O. Raunio and L. Turakka, Pharm. Ind., 44,750 (1982).
25- H. Krasowska, Ibid, 40,1381 (1978).

26- D. H. Doshi, W. R. Ravis and G. V. Betageri, Drug Dev. Ind. Pharm., 23, 1167 (1997).

27- A. A. Kassem, S. A. Zaki, N. M. Mursi and S. A. Tayel, Pharm. Ind., 41, 390 (1979).

28- Y. Chiba, N. Kohri, K. Iseki and K. Miyazaki, Chem. Pharm. Bull., 39, 2158 (1991).

29- H. Sekikawa, J. Fujiwara, T. Naganuma, M. Nakano and T. Arita, Ibid, 26, 3033 (1978).

30- H. Suzuki and H. Sunada, Ibid, 45, 1688 (1997).

31- M. Yoshioka, B. C. Hancock and G. Zografi, J. Pharm. Sci., 84, 983 (1995).

32- S. Lynne and G. Zografi, Spectroscopic Pharm. Res., 14,1691 (1997).

33- A. M. Al-Obaid and M. S. Mian, In: "Analytical Profiles of Drug Substances and Excipients", H. G. Brittan (Ed.), Academic Press New York. 22, (1993), p.433.

34- R. M. Silverstein and F. X. Webster, "Spectrometric Identification of Organic Compounds", ( $6^{\text {th }}$ edition), John Wiley \& Sons; New York, (1998), p. 76

35- V. Tantishaiyakul, N. Kaewnopparat and S. Ingkatawornwong, Int. J. Pharm., 143, 59 (1996).

36- Y. E. Hamza, O. A. Sammour and H. A. Abdel-Latif, Pharm. Ind., 56, 286 (1994).

37- G. Van den Mooter, P. Augustijns, N. Blaton and R. Kinget, Int. J. Pharm, 164, 67 (1998).

38- N. M. Najib and M. S. Suleiman, Ibid, 51, 225 (1989).

39- A. A. Al-Angary, G. M. Al-Mahrouk and M. A. Al-Meshal, Pharm. Ind, 58, 260 (1996).

40- H. Suzuki, N. Miyamoto, T. Masada, E. Hayakawa and K. Ito, Chem. Pharm. Bull., 44, 364 (1996).

41- N. M. Najib, M. Suleiman and A. Malakh, Int. J. Pharm., 32, 229 (1986).

42- A. P. Simonelli, M. M. Meshali, A. H. Abd El-Gawad, H. M. Abdel-Aleem and K. E. Gabr, Drug Dev. Ind. Pharm., 20, 2741 (1994).

43- S. H. Khidr, Bull. Pharm. Sci., Assiut University, 17, 81 (1994). 
44- H. Sekikawa, M. Nakano and T. Arita, Chem. Pharm. Bull., 27, 1223 (1979).

45- N. Yagi, Y. Terashima, H. Kenmotsu, H. Sekikawa and M. Takada, Ibid, 44, 241 (1996).

46- M. C. Tros de Ilarduya, C., Martin, M. M. Goni and M.C. Martinez-Oharriz, Drug Dev. Ind. Pharm., 24, 295 (1998).
47- I. Delneuville, J. P. Dechesne and L. Delattre, Int. J. Pharm., 168, 109 (1998).

48- N. M. Najib, M. A. El-Hinnawi and M. S. Suleiman, Ibid, 45, 139 (1988). 